\title{
Fuzzy Logic and Zadeh Algebra
}

\section{Paavo Kukkurainen}

School of Engineering Science, Lappeenranta University of Technology, Lappeenranta, Finland

Email: paavo.kukkurainen@lut.fi

How to cite this paper: Kukkurainen, $\mathrm{P}$. (2017) Fuzzy Logic and Zadeh Algebra. Advances in Pure Mathematics, 7, 353-365. https://doi.org/10.4236/apm.2017.77022

Received: May 22, 2017

Accepted: July 4, 2017

Published: July 7, 2017

Copyright (c) 2017 by author and Scientific Research Publishing Inc. This work is licensed under the Creative Commons Attribution International License (CC BY 4.0).

http://creativecommons.org/licenses/by/4.0/

\begin{abstract}
In this work we create a connection between AFS (Axiomatic Fuzzy Sets) fuzzy logic systems and Zadeh algebra. Beginning with simple concepts we construct fuzzy logic concepts. Simple concepts can be interpreted semantically. The membership functions of fuzzy concepts form chains which satisfy Zadeh algebra axioms. These chains are based on important relationship condition (1) represented in the introduction where the binary relation $R_{m}$ of a simple concept $m$ is defined more general in Definition 2.10. Then every chain of membership functions forms a Zadeh algebra. It demands a lot of preliminaries before we obtain this desired result.
\end{abstract}

\section{Keywords}

AFS Fuzzy Logic System, Zadeh Algebra, Simple Concepts,

Membership Functions

\section{Introduction}

Starting with simple concepts such as "young people" or "tall people" it is possible to form AFS logic system $\left((E M, \vee, \wedge,)^{\prime}\right)$. The elements are fuzzy concepts constructed by simple concepts. Notice that $(E M, \vee, \wedge)$ is a completely distributive lattice and is called the $E I$ (expanding one set $M$ ) algebra over $M$. So the AFS logic system is a completely distributive lattice equipped with the logical negation '. Let $X$ be a non-empty set. For any $\zeta \in E M$, let $\mu_{\zeta}: X \rightarrow[0,1]$ be a membership function of the concept $\zeta$. Moreover, we assume that all the elements $\mu_{\zeta}$ in the set $\left\{\mu_{\zeta} \mid \zeta \in E M\right\}$ satisfy the three conditions, Definition 2.17. Consider a binary relation $R_{\zeta} \in X \times X$ of the concept $\zeta$. For example, for any two persons $x$ and $y,(x, y) \in R_{\zeta}$ if and only if

$$
R_{\zeta}=\left\{(x, y) \mid(x, y) \in X \times X, \text { age }_{x} \geq \text { age }_{y}\right\}
$$

where $\zeta$ is a fuzzy concept "old". The exact definition is represented in 
Definition 2.10. In Section 2 all the results are known and can be found from [1]. Also the used examples are there. For Zadeh algebra axioms we refer to [2]. In Section 3 it is proved new results. But all the preliminaries represented in Section 2 are necessary to know for understanding these results and their proofs. The crucial condition is

$$
(x, z) \in R_{m} \Rightarrow(y, x) \in R_{m}
$$

for simple concepts $m$ and for all the pairs $(x, z)$ and $(y, x)$ in $X \times X$. In fact, the condition determines a chain $(\zeta)_{\zeta \in E M}$, Lemma 3.1 (c). Let

$$
\left\{\mu_{\zeta} \mid \mu_{\zeta}: X \rightarrow[0,1]\right\}
$$

be a set of membership functions of the concept $\zeta$ of the AFS fuzzy logic system $\left(E M, \vee, \wedge,{ }^{\prime}\right)$. According to Proposition 3.4 a chain $\left(\mu_{\zeta}\right)_{\zeta \in E M}$ corresponding to the chain $(\zeta)_{\zeta \in E M}$ satisfies the seven Zadeh algebra axioms and then forms some Zadeh algebra, Proposition 3.5. These are the two main results. Observing that by Lemma 3.1 (a) the condition (1) implies the condition (2) needed in Proposition 3.4.

$$
(x, z) \in R_{m} \Rightarrow(y, z) \in R_{m}
$$

In the conclusion it is illustrated the research motivation and contribution of this paper.

\section{Preliminaries}

\subsection{Lattices}

In this subsection we refer to [3], pages $1,2,6,8,9,10,119$ and [1], pages 61-64, $67,77$.

Definition 2.1. A partially ordered set or a poset is a set in which a binary relation $\leq$ is defined satisfying the following conditions $(\mathrm{P} 1)-(\mathrm{P} 3)$ :

(P1) For all $x, x \leq x$.

(P2) If $x \leq y$ and $y \leq x$, then $x=y$.

(P3) $x \leq y$ and $y \leq z$, then $x \leq z$.

Let

(P4) Given $x$ and $y$, either $x \leq y$ or $y \leq x$.

A poset which satisfies (P4) is said to be linearly ordered and is called a chain.

Let $X$ be a subset of a poset $P$. Denote the least upper bound of $X$ by l.u.b. i.e. $\sup X$ and the greatest lower bound of $X$ by g.l.b. i.e. inf $X$.

Definition 2.2. A lattice $L$ is a poset $P$ where any two of whose elements $x$ and $y$ have g.l.b. or a meet denoted by $x \wedge y$, and l.u.b. or a join denoted by $x \vee y$. A lattice $L$ is complete if each of its subsets has l.u.b. and g.l.b. in $L$.

It is clear that any nonvoid complete lattice contains a least element 0 and a greatest element 1.

In any lattice $L$ (or a poset), the operations $\wedge$ and $\vee$ satisfy the following laws, whenever the expressions are refered to exist: 
(L1) $x \wedge x=x, x \vee x=x$

(L2) $x \wedge y=y \wedge x, x \vee y=y \vee x$

(L3) $x \wedge(y \wedge z)=(x \wedge y) \wedge z, x \vee(y \vee z)=(x \vee y) \vee z$

(L4) $x \wedge(x \vee y)=x \vee(x \wedge y)=x$

Conversely, any system $L$ with the two binary operations satisfying (L1) (L4) is a lattice.

Moreover, $x \leq y$ is equivalent to each of the conditions

$$
x \wedge y=x \text { and } x \vee y=y
$$

If a poset $P$ (or a lattice) has an 0 , then $0 \wedge x=0$ and $0 \vee x=x$ for all $x \in P$. If $P$ has a universal upper bound $I$, then $x \wedge I=x$ and $x \vee I=I$ for all $x \in P$.

Definition 2.3. A lattice $L$ is distributive if and only if the conditions

$$
\begin{aligned}
& x \wedge(y \vee z)=(x \wedge y) \vee(x \wedge z) \\
& x \vee(y \wedge z)=(x \vee y) \wedge(x \vee z)
\end{aligned}
$$

hold in $L$. In fact, these conditions are equivalent if they are valid.

Definition 2.4. [1], pages 77, 116 or [3], page 119

Let $L$ be a complete lattice. Then $L$ is called a completely distributive lattice if it satisfies the extended distributive laws: for any family $\left\{a_{i j} \in L \mid i \in I, j \in J_{i}\right\}$ where $I$ and $J_{i}$ are non-empty indexing sets, the following equations are valid

$$
\begin{aligned}
& \widehat{\wedge}_{i \in I}\left(\bigvee_{j \in J_{i}} a_{i j}\right)=\underset{f \in \prod_{i \in I}}{\bigvee}\left(\widehat{\wedge}_{i \in I} a_{i f(i)}\right) \\
& \bigvee_{i \in I}\left(\bigwedge_{j \in J_{i}} a_{i j}\right)=\bigwedge_{f \in \prod_{i \in I} J_{i}}\left(\bigvee_{i \in I} a_{i f(i)}\right)
\end{aligned}
$$

\subsection{A Survey to Simple Concepts and Their Operations}

In this subsection we approach to simple concepts and their operations because it is necessary to form the idea what do simple concepts mean. The exact definition will be represented in Definition 2.13. All these are based on [1], pages 113,114 .

Consider the set of four people $x_{1}, x_{2}, x_{3}, x_{4}$ and a simple concept "hair colour". By intuition, we may set: $x_{1}$ has "hair black" with number 6 and $x_{2}, x_{3}, x_{4}$ with numbers $4,6,3$. So, the numbers imply the order $x_{4}>x_{2}>x_{3}=x_{1}$ which can be interpreted as follows: Moving from right to left, the relationship states how strongly the hair colour resembles black colour. More exactly, $x_{i}>x_{j}$ means that the hair of $x_{i}$ is closer to the black colour than the colour of the hair which $x_{j}$ has.

Let $M$ be a set of fuzzy or Boolean concepts on the set $X$. For each $m \in M$ we associate to a single feature. For example $m_{1}$ : "old people" is a fuzzy concept but $m_{2}$ : "male" is a Boolean concept. In fact, $M$ is a set of simple concepts. In general let $A \subseteq M$ and denote by $\prod_{m \in A} m$ a conjugation of the 
concepts $m$ on $A$. Correspondingly $\sum_{m \in A} m$ means a disjunction.

Example 2.5. Let $m_{1}$ : "old people", $m_{2}$ : "male", $m_{3}$ : "tall people". Then $m_{1} m_{2}$ : "old males" and $m_{1}+m_{3}$ : "old or tall people". Further,

$m_{1} m_{2}+m_{2} m_{3}+m_{1} m_{2} m_{3}$ : “old or tall males". However, $m_{1} m_{2}+m_{2} m_{3}$ means the same. This is because for any person $x$ the degree of $x$ belonging to the fuzzy concept represented by $m_{1} m_{2} m_{3}$ is always less than or equal to the degree of $x$ belonging to the fuzzy concept represented by $m_{1} m_{2}$ or $m_{2} m_{3}$. Therefore the former $m_{1} m_{2} m_{3}$ is including in both of the latter ones $m_{1} m_{2}$ or $m_{2} m_{3}$.

\subsection{AFS Fuzzy Logic System}

All the definitions and the propositions with their proofs are represented in [1], pages 115-123. For a moment we give up the assumption that $M$ consists only of simple concepts. Let $M$ be a non-empty set. The set $E M^{\star}$ is defined by

$$
E M^{\star}=\left\{\sum_{i \in I}\left(\prod_{m \in A_{i}} m\right) \mid A_{i} \subseteq M, i \in I, I \text { is any non-empty indexing set }\right\}
$$

where the elements of $E M^{\star}$ are expressed semantically with "equivalent to", "or" (disjunction) and "and" (conjunction).

Definition 2.6. Let $\mathrm{M}$ be a non-empty set. A binary relation $\mathrm{R}$ on $E M^{\star}$ is defined as follows: for

$$
\begin{gathered}
\sum_{i \in I}\left(\prod_{m \in A_{i}} m\right), \sum_{j \in J}\left(\prod_{m \in B_{j}} m\right) \in E M^{\star}, \\
{\left[\sum_{i \in I}\left(\prod_{m \in A_{i}} m\right)\right] R\left[\sum_{j \in J}\left(\prod_{m \in B_{j}} m\right)\right] \Leftrightarrow}
\end{gathered}
$$

(1) $\forall A_{i}, \quad i \in I, \exists B_{h}, h \in J$ such that $A_{i} \supseteq B_{h}$,

(2) $\forall B_{j}, j \in J, \exists A_{k}, k \in I$ such that $B_{j} \supseteq A_{k}$.

$R$ is an equivalence relation and we define $E M$ as the quotient set $E M^{\star} / R$.

Proposition 2.7. Let $M$ be a non-empty set. Then $(E M, \vee, \wedge)$ forms a completely distributive lattice under the binary compositions $\vee$ and $\wedge$ defined as follows: for any

$$
\begin{gathered}
\sum_{i \in I}\left(\prod_{m \in A_{i}} m\right), \sum_{j \in J}\left(\prod_{m \in B_{j}} m\right) \in E M, \\
\sum_{i \in I}\left(\prod_{m \in A_{i}} m\right) \vee \sum_{j \in J}\left(\prod_{m \in B_{j}} m\right)=\sum_{k \in I \sqcup J}\left(\prod_{m \in C_{k}} m\right) \triangleq \sum_{i \in I}\left(\prod_{m \in A_{i}} m\right)+\sum_{j \in J}\left(\prod_{m \in B_{j}} m\right) \\
\sum_{i \in I}\left(\prod_{m \in A_{i}} m\right) \wedge \sum_{j \in J}\left(\prod_{m \in B_{j}} m\right)=\sum_{i \in I, j \in J}\left(\prod_{m \in A_{i} \cup B_{j}} m\right)
\end{gathered}
$$

where the disjoint union $I \sqcup J$ means that every element in $I$ and every element in $J$ are always regarded as different elements in $I \sqcup J$. Therefore for any $k \in I \sqcup J, C_{k}=A_{k}$ if $k \in I$, and $C_{k}=B_{k}$ if $k \in J$.

The proof of the proposition can be found from [1].

To be a distributive lattice means that for any $\gamma, \zeta, \eta \in E M$ 


$$
\begin{aligned}
& \gamma \wedge(\zeta \vee \eta)=(\gamma \wedge \zeta) \vee(\gamma \wedge \eta) \\
& \gamma \vee(\zeta \wedge \eta)=(\gamma \vee \zeta) \wedge(\gamma \vee \eta)
\end{aligned}
$$

A completely distributive lattice is defined in Definition 2.4. Because $(E M, \vee, \wedge)$ is such a lattice it guarantees the existance of the $E M$ elements $\sum_{i \in I}\left(\prod_{m \in A_{i}} m\right)$ and $\sum_{j \in J}\left(\prod_{m \in B_{j}} m\right)$. We can also define the order in $(E M, \vee, \wedge)$ as follows:

$$
\sum_{i \in I}\left(\prod_{m \in A_{i}} m\right) \geq \sum_{j \in J}\left(\prod_{m \in B_{j}} m\right) \Leftrightarrow \forall B_{j}, j \in J, \exists A_{k}, k \in I \text { such that } B_{j} \supseteq A_{k} .
$$

Further, as a (distributive) completely lattice $(E M, \vee, \wedge)$ is also a complete lattice.

The lattice $(E M, \vee, \wedge)$ is called the $E I$ (expanding one set $M$ ) algebra over $M$.

Proposition 2.8. Let $M$ be a set and $g: M \rightarrow M$ be a map satisfying $g(g(m))=m$ for all $m \in M$. If the operator ${ }^{g}: E M \rightarrow E M$ is defined as follows

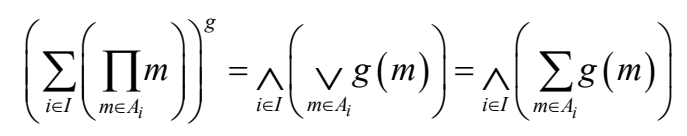

Then for any $\alpha, \beta \in E M, g$ has the following properties:

(1) $\left(\alpha^{g}\right)^{g}=\alpha$,

(2) $(\alpha \vee \beta)^{g}=\alpha^{g} \wedge \beta^{g},(\alpha \wedge \beta)^{g}=\alpha^{g} \vee \beta^{g}$,

(3) $\alpha \leq \beta \Rightarrow \alpha^{g} \geq \beta^{g}$

Therefore the operator ${ }^{g}$ is an order reversing involution in the $E I$ algebra $(E M, \vee, \wedge)$.

The operator ${ }^{g}$ defines the negation $m^{\prime}$ of the concept $m: m^{\prime}=g(m)$. Then $m^{\prime \prime}=(g(m))^{\prime}=g(g(m))=m$.

Let $\zeta=\sum_{i \in I}\left(\prod_{m \in A_{i}} m\right) \in E M$. Then

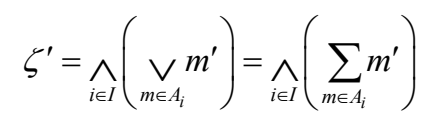

stands for the logical negation of $\zeta \cdot\left(E M, \vee, \wedge,{ }^{\prime}\right)$ is called an AFS fuzzy logic system.

Example 2.9. Let $m_{1}$ : "old people", $m_{2}$ : "tall people", $m_{3}$ : "males". Then

$$
\begin{gathered}
\zeta=m_{1} m_{3}+m_{2}=m_{1} m_{3} \vee m_{2} \\
\zeta^{\prime}=\left(m_{1} m_{3}+m_{2}\right)^{\prime}=\left(m_{1} \wedge m_{3} \vee m_{2}\right)^{\prime}=\left(m_{1}^{\prime} \vee m_{3}^{\prime}\right) \wedge m_{2}^{\prime} \\
=\left(m_{1}^{\prime} \wedge m_{2}^{\prime}\right) \vee\left(m_{3}^{\prime} \wedge m_{2}^{\prime}\right)=m_{1}^{\prime} m_{2}^{\prime}+m_{3}^{\prime} m_{2}^{\prime} .
\end{gathered}
$$

where $\zeta$ : "old males or tall people" and $\zeta^{\prime}$ : "not old and not tall people or not tall males".

The AFS fuzzy logic system $\left(E M, \vee, \wedge,{ }^{\prime}\right)$ can be regarded as a completely 
distributive lattice. It is also a complete lattice. But this lattice is equipped with the logical negation.

We conclude that the complexity of human concepts is a direct result of the combinations of a few relatively simple concepts. In fact, some suitable simple concepts play the same role as used in linear vector spaces and we can regard them as a "basis".

\subsection{Relations, Simple and Complex Concepts}

For this subsection we refer to [1], pages 124, 125.

Definition 2.10. Let $\zeta$ be any concept on the universe of discourse $X$. $R_{\zeta} \subset X \times X$ is called the binary relation of the concept $\zeta$ if $R_{\zeta}$ satisfies: $x, y \in X,(x, y) \in R_{\zeta}$ if and only if $\mathrm{x}$ belongs to concept $\zeta$ at some extent or $x$ is a member of $\zeta$ and the degree of $x$ belonging to $\zeta$ is larger or equal to that of $y$, or $x$ belongs to concept $\zeta$ at some degree and $y$ does not at all.

Example 2.11. Let fuzzy concept $\zeta$ : "old" and

$$
R_{\zeta}=\left\{(x, y) \mid(x, y) \in X \times X, \text { age }_{x} \geq \text { age }_{y}\right\}
$$

Therefore $(x, x) \in R_{\zeta}$ means that $\mathrm{x}$ belongs to $\zeta$ at some degree and that $(x, x) \notin R_{\zeta}$ means that $x$ does not belong to $\zeta$ at all. If for the two persons $x$ and $y$, age $_{x}=30$ and age $_{y}=20$ then $(x, y) \in R_{\zeta}$ but $(y, x) \notin R_{\zeta}$.

Example 2.12. Let fuzzy concept $\zeta$ : "hair black" and define $R_{\zeta}$ in the corresponding way as above. By human intuition, we assume that for the three persons $x_{i}, i=1,2,3$ the degree of $\zeta$ is the following: black $_{x_{1}}>$ black $_{x_{2}}>$ black $_{x_{3}}$ but the fourth person $x_{4}$ has no hairs. Then $\left(x_{1}, x_{2}\right),\left(x_{1}, x_{3}\right),\left(x_{2}, x_{3}\right) \in R_{\zeta}$ and $\left(x_{4}, x_{4}\right) \notin R_{\zeta}$ but $\left(x_{i}, x_{4}\right) \in R_{\zeta}$. See Definition 2.13 (2).

Definition 2.13. Let $X$ be a set and $R$ be a binary relation on $X . R$ is called a sub-preference relation on $X$ if for $x, y, z \in X, x \neq y, R$ satisfies the following conditions:

(1) if $(x, y) \in R$, then $(x, x) \in R$,

(2) if $(x, x) \in R$ and $(y, y) \notin R$, then $(x, y) \in R$,

(3) if $(x, y) \in R$ and $(y, z) \in R$, then $(x, z) \in R$,

(4) if $(x, x) \in R$ and $(y, y) \in R$, then either $(x, y) \in R$ or $(y, x) \in R$.

We define that a concept $\zeta$ on $X$ is simple if $R_{\zeta}$ is a sub-preference relation on $X$. Otherwise $\zeta$ is called a complex concept on $X$.

Example 2.14. Let $\zeta$ : "old people". The concept is simple. For example if for the persons $x_{1}, x_{2}, x_{3}$ we have age $e_{x_{1}}>a g e_{x_{2}}>a g e_{x_{3}} R_{\zeta}$ is a sub-preference relation on the set $\left\{x_{1}, x_{2}, x_{3}\right\}$ where $R_{\zeta}$ is the binary relation defined in Example 2.11. Observe that the latter of the assumptions of (2) in Definition 2.13 is not valid and so the condition (2) is valid. In general it is known that all elements belonging to a simple concept at some degree are comparable and are arranged in a linear order, that is, they form a chain. In above we can think shortly that $x_{1}>x_{2}>x_{3}$.

Further, there exists a pair of different elements belonging to a complex 
concept at some degree such that their degrees in this complex concept are incomparable.

Example 2.15. The set $X$ consists of disjoint sets $Y$ : "males" and $Z$ : "females". The concept $\zeta$ : "beautiful" is simple on $Y$ and on $Z$ :

$$
\begin{aligned}
& R_{\zeta}=\left\{\left(x_{1}, x_{2}\right) \mid\left(x_{1}, x_{2}\right) \in Y \times Y, b_{x_{1}} \geq b_{x_{2}}\right\} \\
& R_{\zeta}=\left\{\left(y_{1}, y_{2}\right) \mid\left(y_{1}, y_{2}\right) \in Z \times Z, b_{y_{1}} \geq b_{y_{2}}\right\}
\end{aligned}
$$

However, if we apply $\zeta$ to the whole set $X$ it is a complex concept because the degrees of the elements $x \in X$ and $y \in X$ may be incomparable:

If $x \in Y$ and $y \in Z$, then $(x, y) \notin R_{\zeta}$ and If $y \in Y$ and $x \in Z$, then $(y, x) \notin R_{\zeta} \quad$ In this case $(x, x) \in R_{\zeta} \quad$ and $\quad(y, y) \in R_{\zeta} \quad$ implies that both $(x, y) \notin R_{\zeta}$ and $(y, x) \notin R_{\zeta}$. The condition (4) in Definition 2.13 is not satisfied and so $\zeta$ is a complex concept.

\subsection{The AFS Fuzzy Logic and Coherence Membership Functions}

For introduction to characteristic and membership functions we refer to [4], page 255, and [5], pages 12-18. Definitions 2.16 and 2.17 can be found from [1], pages 128, 130. We first become acquainted with concepts fuzzy sets and membership functions.

Let $X \neq \varnothing$ and $x \in X, A \subset X$. Define a characteristic function for the set $A$ as follows:

$$
f_{A}: X \rightarrow\{0,1\} \quad f_{A}= \begin{cases}1, & x \in A \\ 0, & \text { otherwise }\end{cases}
$$

Consider an extended case $0 \leq f_{A}(x) \leq 1$, that is, $0<f_{A}(x)<1$ is also possible. We call for the set $A \subset X$

(a) a crisp set, if its characteristic function is $f_{A}: X \rightarrow\{0,1\}$,

(b) a fuzzy set, if its extended characteristic function or a membership function is $\mu_{A}: X \rightarrow[0,1]$.

Therefore for every element $x \in X$ there is a membership degree $\mu_{A}(x) \in[0,1]$. The set of pairs

$$
A=\left\{\left(x, \mu_{A}(x)\right) \mid x \in X\right\}
$$

determines completely the fuzzy set $A$. The characteristic function of a crisp set $A$ is a special case of a membership function $\mu_{A}: X \rightarrow[0,1]$.

Definition 2.16. [1] Let $X, M$ be sets and $2^{M}$ be the power set of $M$. Let $\tau: X \times X \rightarrow 2^{M} .(M, \tau, X)$ is called an AFS structure if $\tau$ satisfies the following axioms:

(1) $\forall\left(x_{1}, x_{2}\right) \in X \times X, \tau\left(x_{1}, x_{2}\right) \subseteq \tau\left(x_{1}, x_{1}\right)$,

(2) $\forall\left(x_{1}, x_{2}\right),\left(x_{2}, x_{3}\right) \in X \times X, \tau\left(x_{1}, x_{2}\right) \cap \tau\left(x_{2}, x_{3}\right) \subseteq \tau\left(x_{1}, x_{3}\right)$.

We again return to the case that $M$ is a set of simple concepts.

Let $X$ be a set of objects and $M$ be a set of simple concepts on $X$. $\tau: X \times X \rightarrow 2^{M}$ is defined as follows: for any $(x, y) \in X \times X$ 


$$
\tau(x, y)=\left\{m \mid m \in M,(x, y) \in R_{m}\right\} \in 2^{M}
$$

where $R_{m}$ is the binary relation of simple concepts $m \in M$ defined in Definition 2.10 (it was defined more general than for simple concepts).

It is proved in [1] that $(M, \tau, X)$ is an $A F S$ structure.

Definition 2.17. [1] Let $(M, \tau, X)$ be an AFS structure of a data set $X$. For $x \in X, A \subseteq M$, the set $A^{\tau}(x) \subseteq X$ is defined as follows:

$$
A^{\tau}(x)=\{y \mid y \in X, \tau(x, y) \supseteq A\}
$$

For $\zeta \in E M$, let $\mu_{\zeta}: X \rightarrow[0,1]$ be the membership function of the concept $\zeta .\left\{\mu_{\zeta}(x) \mid \zeta \in E M\right\}$ is called a set of coherence membership functions of the $A F S$ fuzzy logic system $\left(E M, \vee, \wedge,{ }^{\prime}\right)$ and the $A F S$ structure $(M, \tau, X)$, if the following conditions are satisfied:

(1) For $\alpha, \beta \in E M$, if $\alpha \leq \beta$ in lattice $\left(E M, \vee, \wedge,{ }^{\prime}\right)$, then $\mu_{\alpha}(x) \leq \mu_{\beta}(x)$ for any $x \in X$.

(2) For $x \in X, \eta=\sum_{i \in I}\left(\prod_{m \in A_{i}} m\right) \in E M$, if $A_{i}^{\tau}(x)=\varnothing$ for all $i \in I$ then $\mu_{\eta}(x)=0$.

(3) For $x, y \in X, A \subseteq M, \eta=\prod_{m \in A} m \in E M$, if $A^{\tau}(x) \subseteq A^{\tau}(y)$, then $\mu_{\eta}(x) \leq \mu_{\eta}(y)$; if $A^{\tau}(x)=X$ then $\mu_{\eta}(x)=1$.

Remark: It is important to see that $M$ consists of simple elements $m$.

\subsection{Zadeh Algebra}

We refer to [2].

Definition 2.18. Suppose that $(L, \vee, \wedge)$ is a complete distributive lattice. Let $L^{X}=\{\mu \mid \mu: X \rightarrow L\}$ be the set of all functions from $X$ to $L$. Assume that the lattice operations the least upper bound $\vee$ and the greatest lower bound $\wedge$ on $L$ are extended pointwise for the functions on $L^{X}$. Further, define the extreme constant functions $X \rightarrow L, \quad \mathbf{0}: x \mapsto \perp$ and $\mathbf{1}: x \mapsto \top$ for all $x \in X$, where $\perp$ and $T$ are the least and the greatest elements of $L$, respectively. A unary operation $\eta$ on $L$ satisfies the involution property for any $a \in L$, and $\eta$ is extended pointwise for the functions on $L^{X}$, i.e., $\eta(\eta(\mu))=\mu$ for any $\mu \in L^{X}$. Then $Z=\left(L^{X}, \vee, \wedge, \eta, \mathbf{0}, \mathbf{1}\right)$ is called Zadeh algebra if it satiesfies the following conditions:

(Z1) The operations $\vee$ and $\wedge$ are commutative on $L^{X}$.

(Z2) The operations $\vee$ and $\wedge$ are associative on $L^{X}$.

(Z3) The operations $\vee$ and $\wedge$ are distributive on $L^{X}$.

(Z4) The neutral elements of the operations $\vee$ and $\wedge$ are $\mathbf{0}$ and $\mathbf{1}$, respectively, i.e., for all $\mu \in L^{X}$ and for all $x \in X,(\mu \vee \mathbf{0})(x)=\mu(x)$ and $(\mu \wedge \mathbf{1})(x)=\mu(x)$.

(Z5) For any function $\mu \in L^{X}$ and for all $x \in X$, there exists $\eta(\mu) \in L^{X}$ such that $\eta(\mathbf{1}(x))=\mathbf{0}(x) \leq \eta(\mu(x)) \leq \eta(\mathbf{0}(x))=\mathbf{1}(x)$, i.e., $\eta$ is order reversing.

(Z6) $\mathbf{0} \neq \mathbf{1}$. 
(Z7) Zadeh algebra fulfils the Kleene condition: for any function $\mu, v \in L^{X}$ and for any $x, y \in X, \mu(x) \wedge \neg \mu(x) \leq v(y) \vee \neg v(y)$, where $\neg \mu$ and $\neg v$ are the logical negations of $\mu$ and $v$, respectively.

\section{Connection between Coherence Membership Functions of the AFS Fuzzy Logic System $\left(E M, \vee, \wedge,{ }^{\prime}\right)$ and Zadeh Algebra}

Lemma 3.1. Let $\sum_{i \in I}\left(\prod_{m \in A_{i}} m\right)$ and $\sum_{j \in J}\left(\prod_{m \in B_{j}} m\right)$ be elements in EM where concepts $m$ are simple and let $X$ be a non-empty set. If every relation $R_{m} \subset X \times X$ satisfies the condition

$$
(x, z) \in R_{m} \Rightarrow(y, x) \in R_{m}
$$

for pairs $(x, z)$ and $(y, x) \in X \times X$, then

(a) $(x, z) \in R_{m} \Rightarrow(y, z) \in R_{m}$

(b) there exists $A_{k}$ in the set $\left\{A_{i} \mid i \in I\right\}$ such that $A_{k} \subseteq B_{j}$ for every $B_{j}$, that is,

$$
\sum_{i \in I}\left(\prod_{m \in A_{i}} m\right) \geq \sum_{j \in J}\left(\prod_{m \in B_{j}} m\right)
$$

(c) Let $\alpha=\sum_{i \in I}\left(\prod_{m \in A_{i}} m\right), \quad \beta=\sum_{j \in J}\left(\prod_{m \in B_{j}} m\right), \delta=\sum_{v \in V}\left(\prod_{m \in C_{v}} m\right), \cdots$ be the elements in $E M$ such that the condition (a) is satisfied for all pairs $(\alpha, \beta),(\beta, \delta), \cdots$. Then $\alpha \geq \beta \geq \delta \geq \cdots$.

Proof. Assume that the condition (1) holds.

(a) Let $(x, z) \in R_{m}$. Then $(y, x) \in R_{m}$. Because $m$ is simple, $R_{m}$ is a subpreference relation and by Definition 2.13 (3) it is transitive. This implies that $(y, z) \in R_{m}$ and (a) is valid.

(b) Let

$$
\begin{gathered}
\tau(x, z)=\left\{m \in A_{i} \mid(x, z) \in R_{m}\right\} \\
\tau(y, x)=\left\{m \in B_{j} \mid(y, x) \in R_{m}\right\}
\end{gathered}
$$

Because $m$ is simple $\tau(x, y)$ and $\tau(y, z)$ are defined.

Because $(x, z) \in R_{m} \Rightarrow(y, x) \in R_{m}$ we conclude that $\tau(x, z) \subseteq \tau(y, x)$ and so there exists $A_{k}$ in the set $\left\{A_{i} \mid i \in I\right\}$ such that for every $B_{j}$ is $A_{k} \subseteq B_{j}$. If there exists $B_{r}$ which does not contain $A_{k}$ then $B_{r} \supseteq \bigcup A_{i}$ does not hold. This is a contradiction. According to discussion after Proposition 2.7 we have

$$
\sum_{i \in I}\left(\prod_{m \in A_{i}} m\right) \geq \sum_{j \in J}\left(\prod_{m \in B_{j}} m\right)
$$

(c) Consider a pair $(\beta, \delta)$, where $\beta=\sum_{j \in J}\left(\prod_{m \in B_{j}} m\right)$ and $\delta=\sum_{v \in V}\left(\prod_{m \in C_{v}} m\right)$. We will prove that $\beta \geq \delta$, that is, 


$$
\sum_{j \in J}\left(\prod_{m \in B_{j}} m\right) \geq \sum_{v \in V}\left(\prod_{m \in C_{v}} m\right)
$$

if $(y, w) \in R_{m} \Rightarrow(v, y) \in R_{m} \quad$ (condition (1)).

Repeating the proof of (b) we obtain the following: Let

$$
\tau(y, w)=\left\{m \in B_{j} \mid(y, w) \in R_{m}\right\}, \quad \tau(v, y)=\left\{m \in C_{v} \mid(v, y) \in R_{m}\right\}
$$

Then $\tau(y, w) \subseteq \tau(v, y)$ and there exists $B_{l}$ in the set $\left\{B_{j} \mid j \in J\right\}$ such that $B_{l} \subseteq C_{v}$ for all $C_{v}$. This proves $\beta \geq \delta$. In the same way we can prove that $\alpha \geq \beta$ for a pair $(\alpha, \beta)$ and then we conclude that $\alpha \geq \beta \geq \delta \geq \cdots$

Lemma 3.2. Let $\zeta=\sum_{i \in I}\left(\prod_{m \in A_{i}} m\right) \in E M$. If the condition

$$
(x, z) \in R_{m} \Rightarrow(y, x) \in R_{m}
$$

holds for every simple con ${ }_{\mathrm{C}}$ ept $m \in A_{i}$ then

$$
\mu_{\zeta}(x) \leq \mu_{\zeta}(y)
$$

where $\mu_{\zeta}: X \rightarrow[0,1]$ is the membership function of the concept $\zeta$.

Proof. Let $m \in M$ be simple concepts and

$$
\begin{aligned}
& \tau(x, z)=\left\{m \in A_{i} \mid(x, z) \in R_{m}\right\} \\
& A_{i}^{\tau}(x)=\left\{z \in X \mid \tau(x, z) \supseteq A_{i}\right\}
\end{aligned}
$$

Assume that the condition

$$
(x, z) \in R_{m} \Rightarrow(y, x) \in R_{m}
$$

holds for every $m \in A_{i}$. By Lemma 3.1 (a)

$$
(x, z) \in R_{m} \Rightarrow(y, z) \in R_{m}
$$

also holds. Then $\tau(x, z) \subseteq \tau(y, z)$ and so

$$
A_{i} \subseteq \tau(x, z) \Rightarrow A_{i} \subseteq \tau(y, z)
$$

It follows that $A_{i}^{\tau}(x) \subseteq A_{i}^{\tau}(y)$.

Let $\zeta_{i}=\prod_{m \in A_{i}} m$. These $\zeta_{i}$ exist because $E M$ is a completely lattice. By Definition 2.17 (3) $\mu_{\zeta_{i}}(x) \leq \mu_{\zeta_{i}}(y)$ for every $i \in I$ where $\mu_{\zeta_{i}}: X \rightarrow[0,1]$ is a membership function. Let $\zeta=\sum_{i \in I}\left(\prod_{m \in A_{i}}\right) m=\sup _{i \in I} \zeta_{i}$. Also $\zeta$ exists and we obtain $\mu_{\zeta}(x) \leq \mu_{\zeta}(y)$

Lemma 3.3. Assume that the binary relations $R_{m} \subset X \times X$ of simple concepts $m$ satisfy the condition

$$
(x, z) \in R_{m} \Rightarrow(y, x) \in R_{m}
$$

for pairs $(x, z),(y, x) \in X \times X$.

Let $\alpha=\sum_{j \in J}\left(\prod_{m \in B_{j}} m\right)$ and $\beta=\sum_{i \in I}\left(\prod_{m \in A_{i}} m\right)$ be elements in $E M$. Then $\alpha \leq \beta$ and membership functions $\mu_{\alpha}$ and $\mu_{\beta}$ satisfy the Kleene condition

$$
\mu_{\alpha}(x) \wedge \neg \mu_{\alpha}(x) \leq \mu_{\beta}(y) \vee \neg \mu_{\beta}(y)
$$

Proof. By Lemma 3.1 (b), $\quad \alpha \leq \beta$. On the other hand, by Lemma 3.2 


$$
\mu_{\beta}(x) \leq \mu_{\beta}(y)
$$

Because $\alpha \leq \beta$, by Definition 2.17 (1), $\mu_{\alpha}(x) \leq \mu_{\beta}(x)$. We obtain

$$
\mu_{\beta}(y) \vee \neg \mu_{\beta}(y) \geq \mu_{\beta}(y) \geq \mu_{\beta}(x) \geq \mu_{\alpha}(x) \geq \mu_{\alpha}(x) \wedge \neg \mu_{\alpha}(x)
$$

Proposition 3.4. Let

$$
[0,1]^{X}=\left\{\mu_{\zeta} \mid \mu_{\zeta}: X \rightarrow[0,1]\right\}
$$

be a set of membership functions of the AFS fuzzy logic system $(E M, \vee, \wedge, ')$. The EM elements $\zeta$ are of the form

$$
\zeta=\sum_{i \in I}\left(\prod_{m \in A_{i}} m\right)
$$

Let $R_{m}$ be binary relations of simple concepts $m$. If the condition

$$
(x, z) \in R_{m} \Rightarrow(y, z) \in R_{m}
$$

is valid for every simple concept $m \in A_{i}, i \in I$ and $x, y, z \in X$ then the membership functions $\mu_{\zeta}$ satisfy the conditions (Z1) - (Z7) of Zadeh algebra in Definition 2.18.

Proof. We verify the Zadeh algebra axioms: The first three axioms (Z1) - (Z3) are clear.

(Z1) The operations $\vee$ and $\wedge$ are commutative on $[0,1]^{X}$.

$$
\begin{aligned}
& \mu_{\zeta_{1}}(x) \vee \mu_{\zeta_{2}}(x)=\mu_{\zeta_{2}}(x) \vee \mu_{\zeta_{1}}(x) \\
& \mu_{\zeta_{1}}(x) \wedge \mu_{\zeta_{2}}(x)=\mu_{\zeta_{2}}(x) \wedge \mu_{\zeta_{1}}(x)
\end{aligned}
$$

(Z2) The operations $\vee$ and $\wedge$ are associative on $[0,1]^{X}$.

(Z3) The operations $\vee$ and $\wedge$ are distributive on $[0,1]^{X}$.

(Z4) The neutral elements of the operations $\vee$ and $\wedge$ are $\mu_{0}: X \rightarrow[0,1]$, $\mu_{0}(x) \equiv 0$ and $\mu_{1}: X \rightarrow[0,1], \mu_{1}(x) \equiv 1$.

$$
\begin{gathered}
\mu_{\zeta}(x)=0 \vee \mu_{\zeta}(x)=\mu_{0}(x) \vee \mu_{\zeta}(x)=\left(\mu_{0} \vee \mu_{\zeta}\right)(x) \\
\mu_{\zeta}(x)=1 \wedge \mu_{\zeta}(x)=\mu_{1}(x) \wedge \mu_{\zeta}(x)=\left(\mu_{1} \wedge \mu_{\zeta}\right)(x)
\end{gathered}
$$

(Z5) For any $\mu_{\zeta}: X \rightarrow[0,1]$ and for all $x \in X$ there exists a unary operation

$$
\eta:[0,1]^{X} \rightarrow[0,1]^{X}, \eta\left(\mu_{\zeta}\right)=\mu_{g(\zeta)}
$$

where the operation $g: E M \rightarrow E M$ is defined by

$$
\left(\sum_{i \in I}\left(\prod_{m \in A_{i}} m\right)\right)^{g}=\bigwedge_{i \in I}\left(\sum_{i \in A_{i}} g(m)\right)
$$

Let $m \in M$ be simple concepts. Observe that we need this assumption for Definition 2.17 used bellow: in Definition 2.16 and Definition 2.17 the definition of $\tau(x, y)$ demands $m$ to be simple. According to Proposition $2.8 \mathrm{~g}$ is an order reversing involution and $g(g(m))=m$ but in this case $m$ need not be simple. We obtain

$$
\eta\left(\eta\left(\mu_{\zeta}\right)\right)=\eta\left(\mu_{g(\zeta)}\right)=\mu_{g(g(\zeta))}=\mu_{\zeta}
$$


Therefore $\eta$ is an involution. Here $\zeta$ is not necessary simple.

Let $\alpha \leq \beta$ be elements in $E M$, and since $g$ is order reversing, $g(\alpha) \geq g(\beta)$. Using Definition $2.17(1)$ it is $\mu_{g(\alpha)}(x) \leq \mu_{g(\beta)}(x)$. Therefore

$$
\eta\left(\mu_{\alpha}\right)=\mu_{g(\alpha)} \geq \mu_{g(\beta)}=\eta\left(\mu_{\beta}\right)
$$

and $\eta$ is order reversing.

(Z6) $\mu_{0}(x) \neq \mu_{1}(x)$ for all $x \in X$

(Z7) The Kleene condition. For any function $\beta, \alpha \in[0,1]^{X}$ and for any $x, y \in X$ we have

$$
\mu_{\beta(y)} \vee \neg \mu_{\beta(y)} \geq \mu_{\beta(y)} \geq \mu_{\beta(x)} \geq \mu_{\alpha(x)} \geq \mu_{\alpha(x)} \wedge \neg \mu_{\alpha(x)}
$$

which is proved in Lemma 3.3.

Proposition 3.5. Let

$$
[0,1]^{X}=\left\{\mu_{\zeta} \mid \mu_{\zeta}: X \rightarrow[0,1]\right\}
$$

be a set of membership functions $\mu_{\xi}$ of the AFS fuzzy logic system $\left(E M, \vee, \wedge,{ }^{\prime}\right)$. The $E M$ elements $\zeta$ are of the form

$$
\zeta=\sum_{i \in I}\left(\prod_{m \in A_{i}} m\right)
$$

Let $R_{m}$ be binary relations of simple concepts $m$. If the condition

$$
(x, z) \in R_{m} \Rightarrow(y, x) \in R_{m}
$$

is valid for every simple concept $m \in A_{i}, i \in I$ and $x, y, z \in X$ then

(a) Functions $\mu_{\zeta}$ form a chain corresponding to the chain $(\zeta)_{\zeta \in E M}$.

(b) Any chain $\left(\mu_{\zeta}\right)_{\zeta \in E M}$ constitutes some Zadeh algebra $\left([0,1]^{X}, \vee, \wedge, \eta, \mu_{0}, \mu_{1}\right)$.

Proof. We conclude

(a) By Lemma 3.1 the elements $\zeta$ forms a chain $(\zeta)_{\zeta \in E M}$. By Definition 2.17 (1) $\zeta_{k} \leq \zeta_{l} \Rightarrow \mu_{\zeta_{k}} \leq \mu_{\zeta_{l}}$.

(b) Lemma 3.1 (a) implies that

$$
(x, z) \in R_{m} \Rightarrow(y, z) \in R_{m}
$$

and in Proposition 3.4 it is proved that every chain $\left(\mu_{\zeta}\right)_{\zeta \in E M}$ satisfies (Z1) (Z7).

\section{Conclutions}

Simple concepts form chains. The elements of any chain form a "basis" in AFS fuzzy logic system $(E M, \vee, \wedge, ')$ with operations disjunction $\vee$, conjunction $\wedge$ and the logical negation. The elements are of the form $\sum_{i \in I}\left(\prod_{m \in A_{i}} m\right)$ where simple concepts $m \in A_{i}$ are defined in Definition 2.13 and operations in $\left(E M, \vee, \wedge,,^{\prime}\right)$ are defined in Proposition 2.7. $(E M, \vee, \wedge)$ is a completely distributive lattice. By means of the binary relations $R_{m} \in X \times X$ defined in Definition 2.10 we construct the condition $(x, z) \in R_{m} \Rightarrow(y, x) \in R_{m}$ which implies the condition $(x, z) \in R_{m} \Rightarrow(y, z) \in R_{m}$. Here $X$ is a non-empty set and $x, y, z$ on $X$ and $m$ are simple concepts. Then the conditions consti- 
tute the two things: first, the membership functions $\mu_{\zeta}: X \rightarrow[0,1]$ of the fuzzy concepts $\zeta$ form chains $\left(\mu_{\zeta}\right)_{\zeta \in E M}$ in $\left(E M, \vee, \wedge,{ }^{\prime}\right)$; second, every chain $\left(\mu_{\zeta}\right)_{\zeta \in E M}$ forms a Zadeh algebra. These results are represented in Propositions 3.4 and 3.5 and we can use them as starting points to continue theoretical considerations. The other way to continue the investigations is to utilize directly the conditions above: there are two kinds of successive events. The first one implies the second one or they have no connection. In the latter case the second event only follows the first one although they are independent of each other. In the first case it is possible to apply to the conditions (above) (1) or (2) represented in the introduction.

In Example 2.1, [2], the set $\{\mu \mid \mu: X \rightarrow[0,1]\}$ of membership functions forms a Zadeh algebra. More exactly, if $I=[0,1]$ then $(I, \vee, \wedge)$ is a complete distributive lattice. Further, $I^{X}=\{\mu \mid \mu: X \rightarrow[0,1]\}$ is the set of membership functions. The operations $\vee$ and $\wedge$ are extended pointwise on $I^{X}$. Now $Z=\left\{I^{X}, \vee, \wedge, \neg, \mathbf{0}, \mathbf{1}\right\}$ is a Zadeh algebra with $\mathbf{0}(x)=0, \mathbf{1}(x)=1$, and the logical negation of $\mu$ is $\neg \mu(x)=(1-\mu)(x)$. In this paper we have considered more general membership functions and constructed Zadeh algebras.

\section{References}

[1] Liu, X.D. and Pedrycz, W. (2004) Axiomatic Fuzzy Set Theory and Its Applications. Studies in Fuzziness and Soft Computing 2009, Springer-Verlag, Berlin Heidelberg.

[2] Mattila, J.K. (2012) Zadeh Algebra as the Basis of Lukasiewicz Logics. Lappeenranta University of Technology, Department of Mathematics and Physics.

[3] Birkhoff, G. (1995) Lattice Theory. American Mathematical Society. 3rd Edition, Eight Printing 1995, Colloqium Publications, ISBN 0-8218-1025-1. Printed in the United States of America.

[4] Mattila, J.K. (2002) Sumean logiikan oppikirja. Johdatus sumean matematiikkaan. Kolmas uusittu painos, Dark Oy, ISBN 951-884-300-7, Vantaa.

[5] Negoita, L.V. and Ralescu, D.A. (1975) Applications of Fuzzy Sets to Systems Analysis. Birkhauser Verlag, ISBN 3-7643-0789-7, Basel und Stuttgart.

https://doi.org/10.1007/978-3-0348-5921-9

Scientific Research Publishing

\section{Submit or recommend next manuscript to SCIRP and we will provide best service for you:}

Accepting pre-submission inquiries through Email, Facebook, LinkedIn, Twitter, etc. A wide selection of journals (inclusive of 9 subjects, more than 200 journals)

Providing 24-hour high-quality service

User-friendly online submission system

Fair and swift peer-review system

Efficient typesetting and proofreading procedure

Display of the result of downloads and visits, as well as the number of cited articles

Maximum dissemination of your research work

Submit your manuscript at: http://papersubmission.scirp.org/

Or contact apm@scirp.org 